\title{
Revisiting the optical properties of the FMO protein
}

\author{
Maaike T. W. Milder • Ben Brüggemann • \\ Rienk van Grondelle • Jennifer L. Herek
}

Received: 24 September 2009/Accepted: 24 February 2010/Published online: 13 March 2010

(C) The Author(s) 2010. This article is published with open access at Springerlink.com

\begin{abstract}
We review the optical properties of the FMO complex as found by spectroscopic studies of the $Q_{y}$ band over the last two decades. This article emphasizes the different methods used, both experimental and theoretical, to elucidate the excitonic structure and dynamics of this pigment-protein complex.
\end{abstract}

Keywords FMO complex · Pigment-protein complex · Exciton energy transfer $\cdot$ Spectroscopy

\section{Introduction}

In 1975, Fenna was the first to resolve the X-ray structure of the Fenna-Matthews-Olson (FMO) complex of

Electronic supplementary material The online version of this article (doi:10.1007/s11120-010-9540-1) contains supplementary material, which is available to authorized users.

M. T. W. Milder

AMOLF, Sciencepark 113, 1098 XG Amsterdam,

The Netherlands

e-mail: milder@amolf.nl

B. Brüggemann

Institut für Physik, Humboldt-Universität zu Berlin,

Newtonstrasse 15, 12489 Berlin, Germany

R. van Grondelle

Department of Physics \& Astronomy, Vrije Universiteit

Amsterdam, de Boelelaan 1081, 1081 HV Amsterdam,

The Netherlands

\section{J. L. Herek ( $\square)$}

Optical Sciences Group, Department of Science and Technology, MESA+ Institute for Nanotechnology, University of Twente, 7500 AE Enschede, The Netherlands

e-mail: j.1.herek@tnw.utwente.nl
Prosthecochloris aestuarii. In photosynthetic membranes of green sulfur bacteria, this protein channels the excitations from the chlorosomes to the reaction center. Since it was the first photosynthetic antenna complex of which the $\mathrm{X}$-ray structure became available, it triggered a wide variety of studies of spectroscopic and theoretical nature, and it therefore has become one of the most widely studied and well-characterized pigment-protein complexes. Owing to its relatively simple structure amongst the light-harvesting complexes, with only seven interacting bacteriochlorophyll a (BChl a) molecules, and with the level of sophistication at which the optical properties are known, it comes as no surprise that the FMO complex serves as a guinea pig for new and ever-improving simulation methods as well as new optical techniques. Remarkably, FMO is still a subject of active investigation and new insights continue to emerge. Even fundamental properties, such as the pigment-protein ratio, remain controversial. The goal of this article is to guide the reader through the mass of information that has appeared over the last $\sim 20$ years on the optical properties of the FMO complex. We attempt to provide an objective view of the experimental data and the parameters and methods used in simulations. Also, where applicable, it is indicated which data and parameter sets have become most favored and for which reasons. In order to keep this article insightful and focused, it is restricted to a discussion of the spectral structure of the $Q_{y}$ transition band of a BChl a molecule at $800 \mathrm{~nm}$. This article will specifically address optical properties of the FMO protein from the most thoroughly characterized green sulfur bacterium Prosthecochloris aestuarii. Similar data on the FMO protein from Chlorobium tepidum can be found in the electronic supplementary material. We hope that this article will enable researchers applying novel, complex techniques to gain an overview of the current understanding of 
the optical properties and photosynthetic processes in the FMO protein.

\section{Structure}

In 1962, John Olson isolated a water-soluble bacteriochlorophyll (BChl a) protein $(150 \mathrm{kDa})$ from green sulfur bacteria (Olson and Romano 1962). This specific protein is part of the light-harvesting system in green sulfur bacteria where it acts as a subantenna to collect sunlight and transfer excitation energy from the light-harvesting antennas to the reaction center. Absorption spectroscopy on extracts of strains of Chlorobium showed that the newly discovered protein contained only $\mathrm{BChl}$ a chromophores, non-covalently bound to a protein envelope (Fig. 1). In 1975, Roger Fenna and Brian Matthews resolved the X-ray structure of the FMO protein from Prosthecochloris aestuarii at $2.8 \AA$ resolution and found that the complex consists of three identical subunits related by $C_{3}$ symmetry, each containing seven $\mathrm{BChl}$ a pigments (Fenna and Matthews 1975). It showed a protein shell in which the BChl a molecules were enclosed. The major part of the outside of the protein shell exposed to the solvent is composed of 15 strands of $\beta$-sheet. The side of the shell that is in contact with one of the other subunits in the trimer consists of four short strands of $\alpha$-helix alternated by regions of the protein without a clear structure. The average distance between BChl a molecules within one subunit of the trimer is $12 \AA$ while the nearest molecule in the neighboring subunit is found at a distance of $24 \AA$. Analysis of the X-ray data showed no evidence for interactions-whether these be covalent or noncovalent-between neighboring $\mathrm{BChl}$ a molecules; however, the same analysis predicted the presence of extensive interactions between the chlorophyll molecules and the protein shell. Besides hydrophobic
Fig. 1 a Representation of the FMO protein trimer of Prosthecochloris aestuarii showing the $\mathrm{BChl}$ a pigments surrounded by the protein envelope. b Protein envelope shell, consisting mainly of $\beta$ sheets, enclosing the seven pigments. $\mathbf{c}$ View of the arrangement of the seven BChl a pigments. Identifier 3eoj [5] in the Brookhaven Protein Databank. Pictures are created with rasmol. The eighth $\mathrm{BChl}$ a is omitted for sake of clarity but can be created using the coordinates from Tronrud et al. (2009)

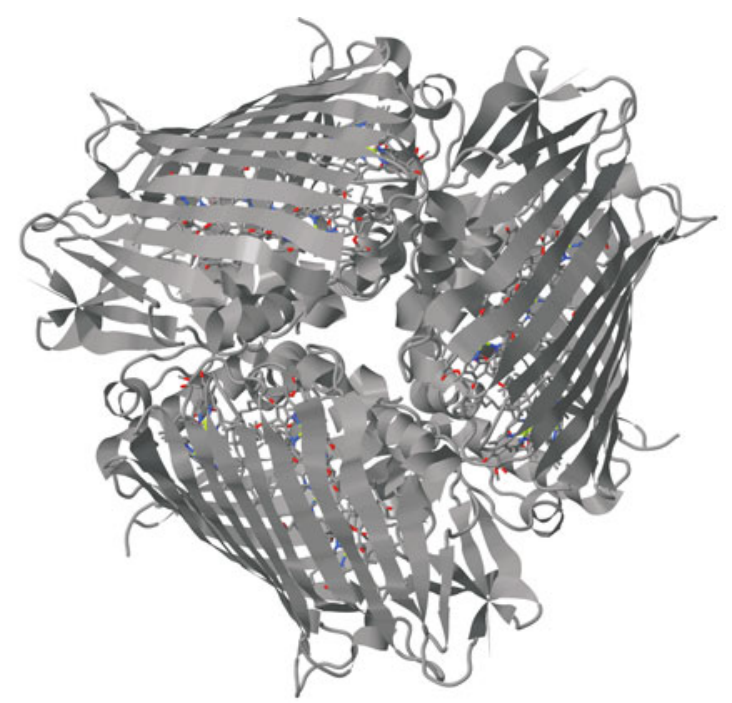

A

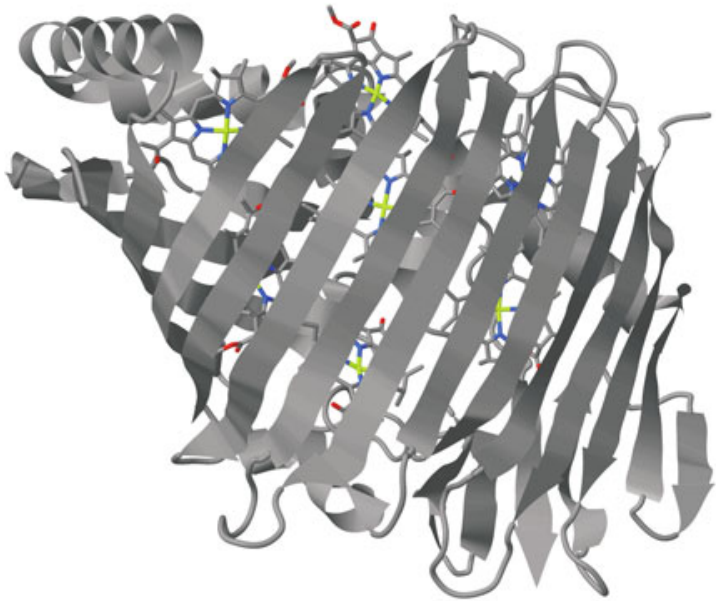

B

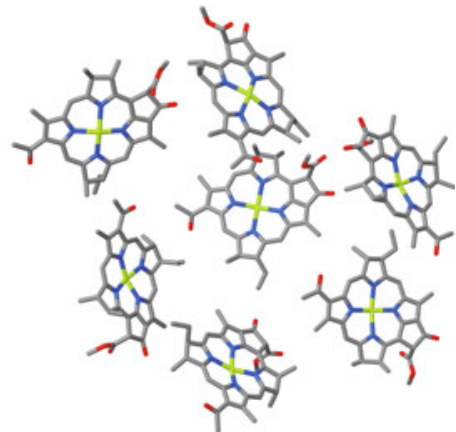

C 
interactions, hydrogen bonding and coordination to the $\mathrm{Mg}$ ion in the BChl a molecule occurs. Over the years, the structure of the FMO protein from Prosthecochloris aestuarii has been refined (Matthews et al. 1979; Tronrud et al. 1986) and recently a $1.3 \AA$ diffraction dataset of the structure has been obtained (Tronrud et al. 2009).

In 1997, the crystal structure of FMO from Chlorobium tepidum was determined at a $2.2 \AA$ resolution ( $\mathrm{Li}$ et al. 1997). Similar to Prosthecochloris aestuarii (Fig. 1a), the distance between the pigments within a subunit range from 4 to $11 \AA$ while the nearest neighbor in a different subunit is over $20 \AA$ away. The $\beta$ sheet is folded in such a way that the strands at the front and the back of the shell are roughly perpendicular to each other (Fig. 1b). The opening in the shell is situated toward the center of the trimer, forming the shape of a shell. The six $\alpha$-helices are located at the open end of the shell and mainly connect the separated $\beta$-strands. BChl a molecules 1 and 2 are situated at the outside of the protein complex, while BChl a 3-7 are located in the center (Fig. 1c). Polar interactions and salt bridges between amino acids insure the formation of a stable trimer. The magnesium ion is a five-coordinate in all the BChl a molecules, although the fifth ligand varies between the pigments. For $\mathrm{BChl} \mathrm{a} \mathrm{1,} \mathrm{3,} \mathrm{4,} \mathrm{6,} \mathrm{and} \mathrm{7,} \mathrm{it} \mathrm{is} \mathrm{a} \mathrm{histidine} \mathrm{residue,} \mathrm{for} \mathrm{BChl} \mathrm{a} \mathrm{5,}$ it is an oxygen atom from a leucine residue, and for $\mathrm{BChl}$ a 2 , the electron density suggests a water molecule as the fifth ligand. The structures of the FMO protein present in the two species Prosthecochloris aestuarii and Chlorobium tepidum show a high degree of similarity (the amino acid sequences are identical to one another within $77 \%$ ). The residues that are not conserved do not alter the interaction between the protein and the $\mathrm{BChl}$ a molecules. Besides that, the relative positions of each of the BChl a molecules in the two species match almost perfectly. The main difference is in the planarity of the tetrapyrrole ring of the $\mathrm{BChl}$ a molecules. For a more detailed description of the comparison between the two species, see Li et al. (1997) and the discussion at the end of this section.

Various spectroscopic investigations using linear absorption spectroscopy, circular dichroism (CD) and linear dichroism (LD) on samples of the isolated FMO protein and the protein associated with membrane vesicles have revealed the orientation of the proteins with respect to the membrane (Melkozernov et al. 1998). The three subunits of the FMO protein are related by $C_{3}$ symmetry and can be modeled as disks, with the axis of the disks parallel to the $C_{3}$ axis (Fig. 2a). The spectroscopic studies show that the $C_{3}$ symmetry axis of the three subunits of the FMO protein is perpendicular to the membrane plane. This implies that the flat sides of the discs is embedded in the membrane (Fig. 2a).

In two recent studies, the presence of an additional BChl a molecule per monomer was proposed. This observation is
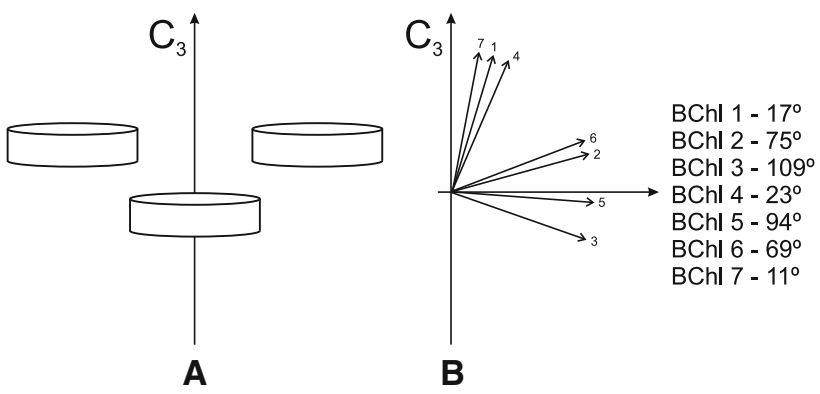

Fig. 2 Orientation of the FMO protein. a The $C_{3}$ axis that relates the three subunits of the FMO protein is parallel to the disc axis and perpendicular to the membrane plane. b The angles between the $Q_{y}$ transitions of the seven $\mathrm{BChl}$ a pigments with respect to the $C_{3}$ axis (Iseri and Gülen 1999)

based on careful studies of high resolution X-ray data. BenShem et al. noticed additional electron density at the interface between the monomers in their newly crystalized and solved structure. They attributed this to the presence of an eighth $\mathrm{BChl}$ a molecule possibly located at the interface between the FMO protein and RC (Ben-Shem et al. 2004). In an attempt to clarify matters, Tronrud et al. (2009) decided to revisit the structure of Chlorobium tepidum as well as collect a new diffraction dataset at $1.3 \AA$ of the FMO protein from Prosthecochloris aestuarii. Their comparison indicated the presence of an eighth BChl a molecule at the same location in both variants, however, with a different local protein structure that could account for the difference in the optical spectra (see "Linear spectra"). The nature of the eighth BChl a molecule is different from the other seven: its occupancy is not unity and it is located in the region of the protein that is directed towards the chlorosome. Its location and the orientation of its transition dipole moment, i.e., parallel to the BChl a in the baseplate, might facilitate energy transfer. In both variants, a carbonyl oxygen binds to the central magnesium atom on one side of the BChl a ring while an $\alpha$-helix covers the other side. It was shown that between the two variants there are three critical differences concerning the amino acid sequence in this helix, close to the additional BChl a molecule. In Prosthecochloris aestuarii at residue 165, threonine is changed into phenylalanine and at residue 168 , alanine is changed into serine. In addition, in the loop that directs the helix back to the protein, an alanine is inserted. These three mutations have the following effect in Prosthecochloris aestuarii: on binding of the eighth $\mathrm{BChl}$ a molecule, the side chain of the Phenylalanine has to move out of the binding pocket. As a result, the $\alpha$-helix moves sufficiently close to the $\mathrm{Mg}$ atom to make an additional link, creating a bidentate interaction between protein and BChl a. However, in Chlorobium tepidum, the smaller Threonine does not move on binding of the BChl; on top of that, the shorter loop of the $\alpha$-helix restricts motion preventing bidentate binding. The 
differences in binding of this extra $\mathrm{BChl}$ a molecule is expected to have a considerable influence on the optical spectra, especially on the CD spectra (vide infra).

\section{Linear spectra}

This section describes the various aspects that come into play on describing and simulating the optical spectra of the FMO complex. First, the differences between the low-temperature absorption spectra of Prosthecochloris aestuarii and Chlorobium tepidum are discussed. This is followed by an account on the site energies of the BChl a molecules. These values cannot be deduced from optical experiments directly and are usually obtained by fits to optical spectra; however, recent attempts to calculate the site energies have emerged. Simulations of the optical spectra are extremely sensitive to the exact choice of site energies, and hence, a detailed overview of the results of different research groups is provided. Subsequently, a third important optical property of the FMO complex is discussed: the pigment with the lowest site energy. This pigment is thought to be the exit pigment of the complex, i.e., the pigment that transfers the excitation energy to the reaction center. As the individual $\mathrm{BChl}$ a molecules interact within the FMO complex, the exciton nature of their excitation is treated and exciton simulations, used to generate various linear spectra, are described. Important parameters in these simulations are the dipolar coupling strength and the linewidth of the transitions. The section ends with a discussion of the controversial nature of the lowest energy absorption band at $825 \mathrm{~nm}$.

Over the years, simulations of the linear spectra have become increasingly sophisticated. Whereas early on, almost all optical properties were hotly debated, in recent times, the tendency is to use parameter sets and methods as obtained and developed by Louwe et al. The validity of their study also extends into the nonlinear regime, as is the topic of the next section.
Absorption spectra at high and low temperatures

The linear absorption spectrum of the FMO complex shows several bands in the wavelength range of 200-900 nm (Olson 2004). The $Q_{y}\left(S_{1}\right)$ absorption band around $800 \mathrm{~nm}$ is the most well-characterized band and the focus of the current study. In membrane factions of Chlorobium tepi$d u m$, this band appears in the spectral region between the absorption band of $\mathrm{BChl} \mathrm{c}$ in the chlorosomes (720$750 \mathrm{~nm}$ ) and the $Q_{y}$ band of the BChl a in the reaction center at $\sim 834 \mathrm{~nm}$ (Melkozernov et al. 1998). The $Q_{y}\left(S_{1}\right)$ absorption band has a temperature-dependent shape. At cryogenic temperatures, in a mixture of Tris buffer and glycerol, the absorption band consists of at least three distinct peaks (Johnson and Small 1991; Gulbinas et al. 1996) (Fig. 3). At elevated temperatures, the fine structure disappears, and the absorption spectrum appears as a broad featureless band.

Low-temperature absorption spectra of the $Q_{y}\left(S_{1}\right)$ band show a clear difference between the FMO complex of Prosthecochloris aestuarii and Chlorobium tepidum; the former has a strong absorption band at $815 \mathrm{~nm}$, while for the latter, the strongest absorption band is at $809 \mathrm{~nm}$. Comparison between the two species with 97\% homology (Chlorobium limicola and Chlorobium tepidum) shows a nearly identical absorption spectrum at $6 \mathrm{~K}$. This indicates that the local protein environment has a limited but observable influence in the spectral differences between the FMO complexes (Francke and Amesz 1997). Li et al. (1997) showed that most ligands of the $\mathrm{BChl}$ a pigments are conserved in both species and propose that the spectral differences find their origin in the non-planarity of the ring in the $\mathrm{BChl}$ a molecules. Recently, Tronrud et al. showed that the difference in absorption spectra of the FMO complex of various green sulfur bacteria can be explained by the structure. As described in the previous section, an additional $\mathrm{BChl}$ a molecule has been observed. Three mutations in the $\alpha$-helix, covering this molecule, lead to a bidentate binding between pigment and protein in the FMO complex from
Fig. 3 Comparison of the lowtemperature absorption spectra of Prosthecochloris aestuarii (triangles) and Chlorobium tepidum (circles) offset by 0.4 for clarity. The figure is adapted from Francke and Amesz (1997) (left). Structure of the BChl a pigment. R represents the phytyl chain. The direction of the $Q_{y}$ transition dipole moment is indicated by the arrow (right)
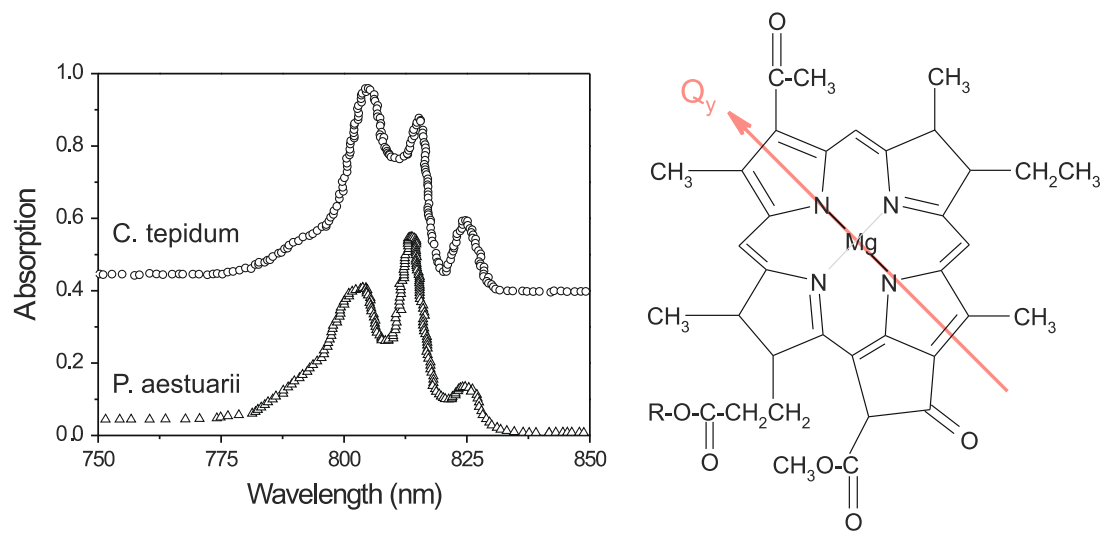
Prosthecochloris aestuarii. As the other seven BChl a molecules are nearly identical, Tronrud et al. ascribe the differences in the spectra to the presence or absence of the additional link to the eighth BChl a molecule. To support this point, a sequence alignment of the FMO protein of several species was performed. This showed that the three mutations, described above, tend to appear together. However, on top of that, the mutations correlate with the type of spectra, i.e., similar to Prosthecochloris aestuarii in the presence of the mutations, and similar to Chlorobium tepidum in the absence of the mutations.

\section{Site energies}

One of the most debated properties of the FMO complex concerns the site energies of the seven BChl a molecules in the complex. These values are needed for exciton calculations of the linear spectra and simulations of dynamics. They are defined as the transition energy of a pigment in the absence of coupling between the pigments. It does, however, depend on local interactions between the BChl a molecule and the protein envelope, and includes electrostatic interactions and ligation. Since the interactions are difficult to identify and even harder to quantify, the site energies are usually treated as independent parameters that are obtained from a simultaneous fit to several optical spectra. Table 1 gives an overview of the different site energies determined by various research groups, using a range of methods described in this section. One of the main differences between the approaches, to obtain the site energies by simulating the spectra, is whether they restrict the interactions to $\mathrm{BChl}$ a molecules within a subunits or wether they include interactions in the whole trimer. These two approaches are labeled in Table 1 with $\mathrm{M}$ (only include interactions within a monomer) and $\mathrm{T}$ (allow interactions between BChl a molecules in the whole trimer).

At the beginning of the 1990s, the optical spectra were fit, assuming interactions between the BChl a pigments from different subunits in one trimer (Johnson and Small 1991; Van Mourik et al. 1994; Rätsep and Freiberg 2007). Although previous efforts to model the system using the full trimer geometry had not been very successful, Pearlstein still expected the $C_{3}$ symmetry of the system to amplify the coupling effect between the intersubunit BChl a molecules (Pearlstein 1992). In contrast to earlier simulations, in his later studies, different site energies were assigned to the 21 transitions. Instead of a single transitions at $802.6 \mathrm{~nm}, 21$ site energies were used as fitting parameters, and the best fit was judged by eye. A mixed approach was employed by Lu et al. and Gülen et al.; the full trimer was taken into account while simultaneously fitting linear optical spectra. However, the same site energies were assigned to the symmetry related $\mathrm{BChl}$ a pigments, resulting in seven adjustable site energies ( $\mathrm{Lu}$ and Pearlstein 1993; Gülen 1996). This approach implies that, although there are only seven different site energies assigned, all the 21 possible exciton transitions in the trimer will be included in the fits (vide infra). Lu and Pearlstein (1993) restricted the interactions to a single subunit and improved the fits from Pearlstein, making use of an algorithm to minimize the difference between the measured and the simulated spectra with various adjustable parameters, amongst which are the seven site energies of the monomer. Their fits were based on two sets of absorption and CD spectra at $77 \mathrm{~K}$, obtained by two different groups (referred to as 1 and 2 in Table 1). A similar

Table 1 Site energies (in $\mathrm{nm}$ ) of $\mathrm{BChl}$ a pigments in the FMO complex of Prosthecochloris aestuarii

\begin{tabular}{|c|c|c|c|c|c|c|c|}
\hline BChl a & 1 & 2 & 3 & 4 & 5 & 6 & 7 \\
\hline Lu and Pearlstein (1993)1 & 784.6 & 798.3 & 800.9 & 803.3 & 799.7 & 811.7 & 822.4 \\
\hline Lu and Pearlstein (1993)2 & 796.8 & 806.9 & 816.9 & 802.2 & 780.2 & 809.3 & 797.2 \\
\hline Gülen (1996) & 804.2 & 802.6 & 805.2 & 806.2 & 807.8 & 815.8 & 803.1 \\
\hline Louwe et al. (1997b) & 811.7 & 804.2 & 824.4 & 811.7 & 795.5 & 803.2 & 804.5 \\
\hline Vulto et al. (1999) & 809.3 & 799.4 & 824.4 & 813.0 & 799.0 & 801.3 & 801.6 \\
\hline Iseri and Gülen (1999) & 808.0 & 802.1 & 822.8 & 809.4 & 795.9 & 800.5 & 804.2 \\
\hline Wendling et al. (2002)1 & 809.7 & 802.2 & 822.4 & 809.7 & 793.7 & 801.3 & 802.6 \\
\hline Wendling et al. (2002)2 & 804.5 & 806.1 & 821.4 & 812.0 & 792.1 & 800.0 & 803.2 \\
\hline Adolphs and Renger (2006)M & 801.6 & 802.6 & 818.0 & 806.1 & 789.6 & 797.1 & 803.9 \\
\hline Adolphs and Renger (2006)T & 803.5 & 803.2 & 817.7 & 809.4 & 788.6 & 796.2 & 799.4 \\
\hline Müh et al. (2007) & 805.8 & 800.1 & 820.1 & 806.8 & 792.4 & 799.5 & 802.7 \\
\hline Adolphs et al. (2008) & 797.1 & 809.1 & 822.4 & 802.9 & 794.3 & 801.9 & 806.1 \\
\hline
\end{tabular}

The annotations $M$ and $T$ stand for simulations taking into account interactions between the seven BChl a molecules in the monomer $(M)$ or between the 21 molecules in the trimer $(T)$

The annotation 1 and 2 represent fits to two datasets from different groups. The annotation $1 *$ and $2 *$ refer to simulations which use different broadening mechanisms 
approach was used by Gülen et al. In contrast to the earlier fits by Pearlstein and Lu et al., CD spectra were excluded from the fits, since they tend to be very sensitive to the experimental conditions like the choice of solvent. Figure $2 b$ shows directions of the individual (not excitonic) transition dipole moments with respect to the $C_{3}$ axis: $\mathrm{BChl}$ a pigments 7,1 , and 4 lie almost parallel to the $C_{3}$ axis, while the orientation of the dipole moments of BChl a 6, 2, 5 , and 3 is almost perpendicular. Gülen used the spatial organization of the individual dipole moments to help restrict and direct the fit. As a start of the fit, the energy of BChl a 6 was fixed between 815 and $820 \mathrm{~nm}$. In order to reproduce the spectra, the site energies of the remaining $\mathrm{BChl}$ a pigments were found to be around $805 \mathrm{~nm}$. Fine tuning of the fits was done by the naked eye.

In contrast to the trimer approach, a different group of researchers fitted the optical spectra, only allowing for interactions within one subunit, the monomer approach. Louwe et al. were among the first to use the monomer approach. Similar to Pearlstein, the site energies were obtained by means of adjusting the parameters manually in the simulations of the spectra, starting from a common site energy at $809.7 \mathrm{~nm}$ (Louwe et al. 1997b). Four possible parameter sets were obtained based on the orientation of the transition dipole moments, as shown previously by Gülen et al. Three of these improved the other existing simulations. However, only one of the basis sets, containing the seven site energies, produced simulations resembling the shape of the spectra (see Table 1). Vulto et al. attempted to simulate the excited state dynamics using the site energies as proposed by Louwe et al. For a satisfactory fit, the site energies needed to be adapted slightly (see Table 1; Vulto et al. 1999). Simulations of both timeresolved and steady-state spectra were the aim of Iseri et al. The site energies were used as free parameters in a manualfitting routine (Iseri and Gülen 1999). As reported in a previos study by Gülen et al., the signs of the bands in the LD spectra limits the choice of site energies as they impose a restriction on the direction of the dipole moments with respect to the $C_{3}$ symmetry axis (see Fig. 2b). An improved fit of absorption and LD spectra was obtained using the site energies as proposed by Louwe et al. and included spectral broadening (vide infra) (Wendling et al. 2002). Further improvements were instigated by a global fit of absorption, CD, and LD spectra. The site energies that were found in these fits are stated in 1, and they are obtained assuming two different types of broadening, denoted by the numbers $1 *$ and $2 *$.

Adolphs and Renger (2006) used a different approach by calculating the "electrochromic shifts" of the site energies by taking into account the interaction between charged amino acids and the pigments. The individual electrochromic shifts were calculated using the Coulomb coupling between the charged amino acids, approximated by point charges, and the difference between the permanent dipole moments of the $\mathrm{BChl}$ a ground and excited state, estimated from Stark experiments. Remarkable is that the red shift of BChl a 3 and the blue shift of BChl a 6 are caused by charged amino acids that are conserved in the structures of Prosthecochloris aestuarii and Chlorobium tepidum. Adolphs et al. show that the fits of the seven site energies for the monomeric and the trimeric structure give similar results. The current method of calculating site energies only succeeded partially in reproducing the site energies obtained from fits to the linear spectra. Therefore, a more elaborate model was needed for better agreement.

Further elaboration, and as a result a good agreement with the experimental spectra, is provided by calculations of the site energies including a detailed description of the charge distribution of both the pigment and the protein states (Müh et al. 2007). Ab initio methods were used to describe the pigments, while a classical electrostatic method was used to describe the whole complex on the atomic level. As a result of the low dielectric constant of water/glycerol below the freezing point, the standard protonation pattern of the amino acids was no longer valid and half of the usually acidic and basic groups turned out to be neutral. This complex method was simplified, without losing the main results by assuming a standard protonation pattern and by the introduction of an effective dielectric constant for screening effects (Adolphs et al. 2008). There exists an earlier account of similar quantum calculations where, amongst others, the effect of the charged amino acids was included (Gudowksa-Nowak et al. 1990). However, the resulting site energies are spread over a range ( 770-840 nm) much larger than what is observed in spectra, hence, and these results are not used for exciton calculations.

While, for some of the earlier calculations and fits, the range of site energies only spans $10 \mathrm{~nm}$, the more recent ones seem to converge to a difference between the highest and lowest site energy of almost $30 \mathrm{~nm}$, which is comparable to the total width of the absorption spectrum. The most widely accepted values of the site energies for Prosthecochloris aestuarii are given by Louwe et al. (see Table 1). Nevertheless, improvements have been obtained using more and more elaborate models and by calculations of the site energies rather than fitting them. In general, only seven different site energies are included as parameters in the fits, however wether or not to include interaction between the monomers remains controversial.

Exit pigment in the FMO complex

The pigment with the lowest site energy is the most likely candidate for an exit pigment, which transfers the 
Table 2 Lowest site energy of the BChls in the FMO complex from Prosthecochloris aestuarii

\begin{tabular}{lll}
\hline References & Site energy (nm) & $\begin{array}{l}\text { Pigment } \\
\text { number }\end{array}$ \\
\hline Pearlstein (1992) & 826.4 & 7 \\
Lu and Pearlstein (1993) & 822.4 & 7 \\
Gülen (1996) & 815.8 & 6 \\
Buck et al. (1997) & 822.5 & 7 \\
Louwe et al. (1997b) & 824.4 & 3 \\
Vulto et al. (1999) & 824.4 & 3 \\
Iseri and Gülen (1999) & 822.8 & 3 \\
Wendling et al. (2002) & $822.4,821.4$ & 3 \\
Adolphs and Renger (2006) & 817.7 & 3 \\
Müh et al. (2007) & 820.1 & 3 \\
Adolphs et al. (2008) & 822.4 & 3 \\
\hline
\end{tabular}

excitation energy from the FMO complex to the reaction center. The position of this pigment within the FMO complex cannot be detected optically because this would require a resolution below the diffraction limit, and, therefore, it can only be assigned from the outcome of exciton simulations. However, since photosynthesis occurs at $300 \mathrm{~K}$, at room temperature, none of the exciton states should be excluded from, a transition dipole-weighted, energy transfer to the reaction core complex. Table 2 shows the different "exit pigments" that have been proposed, with consensus now leaning toward pigment 3. A detailed account on the nature of the electronic state of the exit pigment will be given in "Nature of the lowest energy band".

Pearlstein pioneered the approach of finding the best site energies by looking at absorption, $\mathrm{CD}$, and holeburning spectra, rather than just at spectra from one experimental technique. His fits showed that $\mathrm{BChl}$ a 7 has the lowest site energy (Pearlstein 1992) Subsequently the lowest energy pigment was assigned to be $\mathrm{BChl}$ a 7 or 3 depending on the fitted dataset ( $\mathrm{Lu}$ and Pearlstein 1993). The site energies simultaneously fitted to absorption, LD, and singlet-triplet spectra (Gülen 1996) brought BChl a 6 forward as the pigment with the lowest site energy. It is the best interconnected pigment. Simulations by Buck et al. favored BChl a 7 for that role. They obtained the best fit using parameters deduced from optical spectra of Olson et al. (1976), in which BChl a 7 has the lowest site energy (Buck et al. 1997). By means of fitting new LD and CD data, Louwe et al. (1997b) concluded that the exciton states are mainly localized on one $\mathrm{BChl}$ a and that the lowest energy pigment was $\mathrm{BChl}$ a 3 . This agrees with the results from Stark hole-burning experiments (Rätsep et al. 1998). Since Since then, different theoretical and experimental approaches agree on $\mathrm{BChl}$ a 3 being the pigment with the lowest site energy (Vulto et al. 1999; Iseri and Gülen 1999; Wendling et al. 2002; Adolphs and Renger 2006; Müh et al. 2007; Adolphs et al. 2008). Electron microscopy showed the arrangement of the FMO complex with respect to the reaction center (RC) (Rémigy et al. 1999). The technique lacks the resolution to distinguish between the top and the bottom of the FMO complex. However, from the shape of the FMO complex, it can be deduced that either BChl a 1 and 6 or 3 and 4 form the exit pigments from FMO protein to RC. Wen et al. used mass spectrometry to infer the orientation of the FMO complex with respect to the RC, which is embedded in the cytoplastic membrane (Wen et al. 2009). Their results, in agreement with the theoretical predictions, showed that the BChl a 3 side of the FMO complex interacts with the membrane. Hence, pigment number 3 is the closest to the RC and, therefore, likely to be the exit pigment.

By taking a closer look at the environment of $\mathrm{BChl}$ a 3 , which is generally assumed to have the largest electrochromic shift to lower site energy, a curious arrangement of $\alpha$-helices was observed (Müh et al. 2007). The dipoles of the two helices can be represented by two partial charges on the ends of the helix. The positive and negative partial charges of helix 5 lie in the negative and positive regions, respectively, of the calculated difference $\left(S_{0}-S_{1}\right)$ electrostatic potential. This results in a red shift of the site energies of about $200 \mathrm{~cm}^{-1}$. Similarly, helix 6 gives an additional, however somewhat reduced, redshift of $\sim 100 \mathrm{~cm}^{-1}$. Both helices have a definite effect on the site energies without being dependent on protonation states.

Exciton nature of the BChl a excitations in the FMO protein

The close proximity of the $\mathrm{BChl}$ a molecules $(\sim 10 \AA)$ leads to electronic coupling between them that exceeds the electron-vibrational coupling in the FMO complex. Therefore, the system is usually described by a superposition of the seven molecular BChl a states forming seven exciton states (Van Amerongen et al. 2000), and the electron-vibrational coupling is treated perturbationally. These excitonic interactions $V_{i j}$ between two chromophores $i$ and $j$ are dominated by the relative orientation of the transition dipole moments and the inverse cube of the distance between the $\mathrm{BChl}$ a molecules. The exciton levels have different cross sections and linewidths which together with a close energy spacing results in a dense and complex spectrum. This can be seen in the low-temperature absorption spectra in which only three peaks out of seven are clearly visible.

In order to describe the excitonic wavefunctions in the FMO protein, the following electronic Hamiltonian is used: 
$\hat{H}_{0}=\sum_{j} E_{j}|j\rangle\langle j|+\sum_{j<i} V_{i j}(|j\rangle\langle i|+| i\rangle\langle j|)$

in which $E_{j}$ represents the site energies of the uncoupled BChl a molecules and $|j\rangle$ the corresponding localized excitations. The exciton wavefunctions $|\alpha\rangle$ are obtained by diagonalizing the Hamiltonian as

$|\alpha\rangle=\sum_{j} C_{\alpha}(j)|j\rangle$

using the exciton expansion coefficients $C_{\alpha}(i)$ which represent the contribution of the individual $\mathrm{BChl}$ a molecules to an excitonic transition. The results of such calculations, as performed on the same system by a variety of research groups, are shown in the Tables 3, 4, and 5, where $\alpha$ runs vertically and $i$ horizontally. Pearlstein used a pointmonopole approach to describe the interaction between the individual $\mathrm{BChl}$ a molecules. The transition charge density is calculated for each molecule, represented by point charges at the position of individual atoms, and the interactions of all point charges with those of the other chromophore are considered. All the $21 \mathrm{BChl}$ a of the trimeric FMO complex were included in the model, and the parameters for all the 21 degenerate and non-degenerate exciton transitions are displayed in the original article (Pearlstein 1992). It can be concluded that in each case only one or two of the $\mathrm{BChl}$ a pigments contribute significantly to the squared amplitude of the eigenvectors of the transitions. This means that none of the exciton states is delocalized over the complete subunit, let alone the trimer. Later this was verified by using a similar approach to model the absorption spectra (Gülen 1996). However, the main difference is that now the simpler point-dipole approximation was used to model the interactions between the excitonic transitions of two chromophores by representing the electronic transition of each molecule by a single dipole moment. Later, an approach by the same group followed, which restricted the dipole interactions to one monomer only (Iseri and Gülen 1999).

Table 3 Contribution of the individual $\mathrm{BChl}$ a pigments $j$ to the monomer exciton transitions $\alpha$ in Prosthecochloris aestuarii, occupation probabilities $\left|C_{\alpha}(j)\right|^{2}$ from reference (Gülen 1996)

\begin{tabular}{llllllll}
\hline $\begin{array}{l}\text { Transition } \\
\text { number }\end{array}$ & 1 & 2 & 3 & 4 & 5 & 6 & 7 \\
\hline 1 & 0.004 & 0.001 & 0.004 & 0.082 & 0.340 & 0.510 & 0.059 \\
2 & 0.102 & 0.193 & 0.232 & 0.285 & 0.004 & 0.162 & 0.023 \\
3 & 0.409 & 0.255 & 0.010 & 0.196 & 0.003 & 0.061 & 0.064 \\
4 & 0.017 & 0.017 & 0.186 & 0.005 & 0.160 & 0.003 & 0.613 \\
5 & 0.024 & 0.001 & 0.482 & 0.034 & 0.275 & 0.167 & 0.017 \\
6 & 0.314 & 0.344 & 0.004 & 0.169 & 0.096 & 0.021 & 0.055 \\
7 & 0.130 & 0.189 & 0.081 & 0.229 & 0.122 & 0.076 & 0.169 \\
\hline
\end{tabular}

Results from linear-dichroic absorbance-detected magnetic resonance experiments on FMO at $1.2 \mathrm{~K}$ exhibited similar results as monomeric $\mathrm{BChl}$ a molecules in organic solvents. This technique is sensitive to the triplet state of the complex and, therefore, it was concluded that in FMO, the triplet state is localized on a single $\mathrm{BChl}$ a pigment and not on its delocalized trimeric counterpart (Louwe et al. 1997a). Simultaneous simulation of the spectra obtained from this technique together with $C D$ spectra were performed considering a single subunit only (Louwe et al. 1997b). This approach was justified by the fact that the simulations predict exciton states that are mainly dominated by a single $\mathrm{BChl}$ a, implying that the degree of exciton delocalization is limited in the FMO complex.

Coupling strengths, linewidth, and exciton energies

For exciton simulations of the various spectra (e.g., absorption, LD, CD) of the FMO protein there are three basic ingredients: the site energies, the dipolar coupling (coupling strength), and the optical linewidth. The first is treated in "Site energies", while the latter two will be discussed in this section. The transition dipole strength of BChl a $d\left(D^{2}\right)$ in the FMO complex, has been reported several times in literature. It is an important parameter in simulations of the optical spectra. The values of this dipole strength vary widely and range between 20 and $60 \mathrm{D}^{2}$. Simulations by Pearlstein revealed a dipole coupling strength with a value of $51.6 D^{2}$ (Pearlstein 1992). This value is similar to the one he used in previous calculations and corresponds to the value of $50.8 D^{2}$ used by Fenna. Further successful simulations of steady-state and timeresolved experiments were obtained using values of $51 D^{2}$ (Renger and May 1998) and 30-40 $D^{2}$ (Iseri and Gülen 1999; Wendling et al. 2002). This value was verified by calculations, which resulted in a value of the effective dipole strength of $30 D^{2}$ (Adolphs and Renger 2006) obtained by reducing the dipole strength in vacuum by a factor of 1.25 .

Broadening in optical spectra has two distinct origins, both of which are of importance in the spectroscopic studies of the FMO complex (May and Kühn 2000). The first phenomenon that causes line broadening is static disorder. The seven pigments in the FMO complex all have a slightly different local environment, since the protein envelope that surrounds them differs from pigment to pigment. As a result, there is a different mean energy, center absorption frequency, for each $\mathrm{BChl}$ a. Owing to the differences between, for example, the solvation of all $\mathrm{BChl}$ a 1 pigments in the sample, the center absorption frequency of this pigment is broadened. This effect is referred to as inhomogeneous broadening and can lead to a broad band in the linear absorption spectrum. Inhomogeneous broadening is 
Table 4 Contribution of the individual $\mathrm{BChl}$ a pigments to the monomer exciton transitions in Prosthecochloris aestuarii, occupation amplitudes $C_{\alpha}(j)$ from Louwe et al. (1997b)

\begin{tabular}{lrrrrrrr}
\hline Transition number & \multicolumn{1}{l}{ 1 } & \multicolumn{1}{l}{3} & \multicolumn{1}{l}{4} & \multicolumn{1}{l}{5} & \multicolumn{1}{l}{6} & \multicolumn{1}{l}{7} \\
\hline 1 & -0.066 & -0.116 & 0.955 & 0.259 & 0.035 & 0.027 & 0.042 \\
2 & 0.845 & 0.449 & 0.037 & 0.252 & 0.027 & 0.020 & 0.136 \\
3 & -0.220 & -0.133 & -0.268 & 0.794 & 0.243 & -0.166 & 0.382 \\
4 & 0.015 & -0.143 & -0.111 & 0.348 & -0.293 & 0.818 & -0.300 \\
5 & 0.130 & -0.336 & 0.009 & -0.261 & -0.310 & 0.236 & 0.807 \\
6 & -0.464 & 0.795 & 0.057 & -0.007 & -0.199 & 0.187 & 0.272 \\
7 & -0.018 & 0.043 & 0.014 & -0.223 & 0.847 & 0.459 & 0.139 \\
\hline
\end{tabular}

Table 5 Contribution of the individual BChl a pigments to the monomer exciton transitions in Prosthecochloris aestuarii, occupation probabilities $\left|C_{\alpha}(j)\right|^{2}$ from Iseri and Gülen (1999)

\begin{tabular}{llllllll}
\hline $\begin{array}{l}\text { Transition } \\
\text { number }\end{array}$ & 1 & 2 & 3 & 4 & 5 & 6 & 7 \\
\hline 1 & 0.005 & 0.019 & 0.882 & 0.088 & 0.002 & 0.001 & 0.002 \\
2 & 0.547 & 0.286 & 0.000 & 0.126 & 0.007 & 0.000 & 0.034 \\
3 & 0.090 & 0.052 & 0.094 & 0.490 & 0.091 & 0.042 & 0.141 \\
4 & 0.001 & 0.028 & 0.018 & 0.132 & 0.140 & 0.667 & 0.013 \\
5 & 0.037 & 0.093 & 0.001 & 0.090 & 0.093 & 0.002 & 0.683 \\
6 & 0.319 & 0.520 & 0.003 & 0.000 & 0.051 & 0.016 & 0.091 \\
7 & 0.001 & 0.003 & 0.001 & 0.073 & 0.616 & 0.272 & 0.035 \\
\hline
\end{tabular}

included in the description of optical spectra in two ways: by including a variable linewidth or by introducing one linewidth for all transitions. An example of the first is given by Pearlstein, who employed widths in the range of $\sim 80$ to $\sim 170 \mathrm{~cm}^{-1}$ although there was no physical justification for this large difference (Pearlstein 1992). Exciton simulations by Buck et al. (1997) were performed using $\sim 150 \mathrm{~cm}^{-1}$ for all the transitions in the complex and, therefore, discarded the effect of inhomogeneous broadening shown by Pearlstein to be effective in simulation. Around the same time, linewidths obtained from hole-burning experiments, $\sim 70$ $80 \mathrm{~cm}^{-1}$, were employed by two sets of authors (Gülen 1996; Wendling et al. 2000) to simulate absorption, linear dichroism, singlet-triplet and low-temperature absorption and fluorescence line-narrowing measurements, respectively. Several successful simulations of both steady-state and time-resolved spectra were performed using an inhomogeneous linewidth of $\sim 80 \mathrm{~cm}^{-1}$ (Louwe et al. 1997b; Vulto et al. 1998a, b, 1999).

Besides inhomogeneous broadening, a second physical process that is thought to contribute to broadening of the linewidths is important in the FMO complex. If the changes in the molecular properties are fast compared to the duration of the measurements, then dynamic disorder occurs. This causes homogeneous broadening, because it is equal for the whole system. Initially, hole-burning spectra provided a way to obtain the homogeneous linewidths and revealed values of $\sim 70-80 \mathrm{~cm}^{-1}$ (Johnson and Small 1991). A better description of the spectra was subsequently obtained by fully including the effects of different types of broadening to an existing model proposed earlier by the same authors (Wendling et al. 2002). The two types of broadening were included in simulations of new LD and $\mathrm{CD}$ spectra at low temperatures describing the whole trimer. Inhomogeneous broadening due to the variation in site energies in between subunits and complexes could especially influence the simulations of the polarized spectra. Subsequently, the authors added homogeneous broadening due to dephasing, the lifetimes of the exciton states were calculated using their exciton model. Even without changing the site energies and coupling strengths from reference (Louwe et al. 1997b), the absorption spectra were reproduced better taking broadening into account in the system. The simulations of the LD and CD spectra were further improved by fitting the site energies and the coupling strengths to the experiments using a global fit.

In order to determine the different exciton states and the accompanying transition energy, several approaches were used. To begin with, in the reference (Johnson and Small 1991) exciton energies are determined by simultaneous analysis of different hole-burning spectra. In this case, eight exciton components were observed of which the latter two were assigned to contribute to one band around $825 \mathrm{~nm}$ (vide infra). Pearlstein followed a similar procedure and fitted 21 exciton energies (of which 14 degenerate, see Table 6) to absorption and CD spectra (Pearlstein 1992). There are two more reports on the exciton levels in the trimer, both based on the method described by Pearlstein (Lu and Pearlstein 1993; Gülen 1996). Improvements were made using algorithms to fit the spectra and changing the site wavelengths, which are used to determine the exciton levels, respectively.

In further attempts to model the spectra, only monomers containing seven $\mathrm{BChl}$ a molecules are taken into account (see Table 7). This results in a structure with seven interconnected exciton levels. These simulations require the site energies of the $\mathrm{BChl}$ a molecules as input parameters. Louwe et al. (1997b) set a standard for simulations of the 
Table 6 Exciton energies of Prosthecochloris aestuarii in the trimer in nanometer

\begin{tabular}{llll}
\hline $\begin{array}{l}\text { Exciton } \\
\text { transition }\end{array}$ & $\begin{array}{l}\text { Pearlstein } \\
(1992)\end{array}$ & $\begin{array}{l}\text { Lu and Pearlstein } \\
(1993)^{\mathrm{a}}\end{array}$ & $\begin{array}{l}\text { Gülen } \\
(1996)^{\mathrm{a}}\end{array}$ \\
\hline 1 & 779.7 & 777.7 & 789.63 \\
2,3 & 780.4 & 777.2 & 790.76 \\
4,5 & 789.4 & 787.3 & 792.38 \\
6 & 789.8 & 788.5 & 793.31 \\
7,8 & 797.4 & 797.0 & 801.53 \\
9 & 799.6 & 800.1 & 801.57 \\
10 & 803.8 & 805.1 & 804.10 \\
11,12 & 805.5 & 806.3 & 804.73 \\
13 & 813.0 & 811.6 & 812.50 \\
14,15 & 814.3 & 812.5 & 815.37 \\
16 & 814.7 & 812.8 & 816.46 \\
17,18 & 815.3 & 813.8 & 817.82 \\
19 & 824.1 & 825.0 & 824.80 \\
20,21 & 826.4 & 828.0 & 825.19 \\
\hline
\end{tabular}

${ }^{a}$ The degeneracy of the exciton transitions is different from that proposed by Pearlstein, given in this table, and can be found in the references

Table 7 Exciton energies of Prosthecochloris aestuarii in the monomer

\begin{tabular}{llllll}
\hline Exciton & A & B & C & D & E \\
\hline 1 & $827.1-824.4$ & 825.6 & 825.7 & 825.0 & 823.8 \\
2 & 816.3 & 815.2 & 814.5 & 814.1 & 813.7 \\
3 & 813.0 & 813.5 & 812.2 & 812.8 & 811.5 \\
4 & 807.8 & 806.7 & 805.8 & 805.9 & 804.7 \\
5 & 804.8 & 802.7 & 800.8 & 801.5 & 801.0 \\
6 & 801.3 & 800.2 & 796.4 & 799.6 & 797.8 \\
7 & 793.6 & 791.5 & 793.0 & 791.5 & 789.4 \\
\hline
\end{tabular}

Where A is from Johnson and Small (1991), B is from Louwe et al. (1997b), C is from Vulto et al. (1999), D is from Iseri and Gülen (1999), $\mathrm{E}$ is from Wendling et al. (2002)

spectra. The interaction energies are calculated in the pointdipole approximation assuming a common linewidth for all transitions of $\sim 80 \mathrm{~cm}^{-1}$. Screening by the protein is taken into account by a dielectric constant that was used a global free-fit parameter. The initial calculated dipole strength of $68.9 \mathrm{D}^{2}$ is thus reduced by a factor 2.4 leading to an effective dipole strength of $28.7 D^{2}$, a value that is lower than that proposed by Pearlstein (1992). This value is close to a physically relevant value of the reduced dipole strength in the range of $25-40 D^{2}$. In order to simulate the spectra, a minimum of free parameters was used to fit the essential features of the spectra. The authors proposed that the model can be improved by inclusion of vibrations, lifetime broadening of the highest energy exciton states, and by allowing for different dipole strengths for the individual
$\mathrm{BChl}$ a molecules and a variation of the dielectric constant over the protein. Simulations based on the same exciton model were performed by the following research groups: Vulto et al. (1998a, 1999), Wendling et al. (2000, 2002), and Iseri and Gülen (1999).

Nature of the lowest energy band

The assignment of the bands in the absorption spectrum, especially of the band, the lowest in energy at $825 \mathrm{~nm}$, has proven to be difficult. The number of excitonic states and their respective energies have been the subject of intense debate. Johnson and Small (1991) concluded that lower and higher spectral energy features flanking the hole-burning line can only be explained when excitonic interactions between the BChls are taken into account. Furthermore, the results of spectral hole burning show the presence of eight states. Two of those eight identified exciton states, which have perpendicular symmetry, contribute to this lowest exciton band at $825 \mathrm{~nm}$. Models excluding the interactions between the subunits of the trimer are not successful in describing this experimental data (Johnson and Small 1991). Therefore, Johnson and Small (1991) have developed a model in which this interaction is included leading to a maximum of 14 delocalized states (21 states in total, of which 14 are degenerate). This implies that the $825-\mathrm{nm}$ band comprises of three, slightly shifted, bands of the subunits, of which two are degenerate. For the space group $C_{3}$, the states having E symmetry are degenerate while the states with $\mathrm{A}$ symmetry are not (Atkins 1995). Owing to the varying local environment, there is a certain degree of static disorder in the system leading to an inhomogeneously broadened $825-\mathrm{nm}$ band. The nature of the $825-\mathrm{nm}$ band was confirmed to have a double origin seven years later by means of Stark holeburning studies (Rätsep et al. 1998). However, in this case, the nature of these states was assumed to be much more localized, with the excitons mainly spread over one BChl a molecule. Structural heterogeneity in the complex leads to a variation in the excitation energy of the lowest energy state in the subunits of the trimer. This view was tested by temperature-dependent hole-burning experiments on the FMO protein from Chlorobium tepidum (Rätsep et al. 1999). The 825-nm absorption band was fitted with three Gaussian bands of $\sim 55 \mathrm{~cm}^{-1}$ at $823.0,825.0$, and $827.0 \mathrm{~nm}$, respectively. The dependence of hole width and hole growing kinetics on the burning frequency confirms that there are three bands contributing to the $825-\mathrm{nm}$ band. Triplet minus singlet $(T-S)$ spectra measured by Louwe et al. (1997a) shows that the triplet state is localized on a single BChl a since it demonstrates the same properties as monomeric $\mathrm{BChl}$ a $a$ in organic solvents. The orientations of excitonic transitions in the $Q_{y}$ band were determined relative to the triplet-carrying molecule. In contrast to earlier 
measurements, fluorescence line narrowing experiments showed that the 825-nm absorption band can be accounted for by a single transition in the range of temperature from 4 $\mathrm{K}$ to room temperature (Wendling et al. 2000). This transition is coupled to protein phonons and vibrations in the chromophore. The effect of disorder on the lowest energy band in the trimer was further studied by Monte Carlo simulations (Hayes et al. 2002). The lowest energy band could be fitted with three nearly Gaussian bands of almost identical intensity. One of those band was centered at the absorption maximum of the $825 \mathrm{~nm}$ band, while the maxima of the other two bands where shifted by $\sim-17$ and $\sim+26 \mathrm{~cm}^{-1}$, respectively. Summarizing, the outcome of different experimental techniques do not agree on the nature of the $825 \mathrm{~nm}$ band. While some state that this band is due to a single transition, others include a distribution of the lowest exciton energy in the different subunits of the trimer to account for the observations.

\section{Nonlinear spectra and exciton dynamics in the FMO protein}

This section will discuss both the experimental and theoretical aspects of the time-resolved spectra of the FMO protein. Previously, in "Exciton nature of the BChl a excitations in the FMO protein" and "Coupling strengths, linewidth, and exciton energies", the excitonic structure and simulations of the linear optical spectra were reviewed. Starting from this knowledge, it is a small, yet complex step to simulate the time-dependent behavior of the exciton states. After optical excitation, the population in the exciton states eventually decays back to the ground state. The relaxation pathways and the accompanying time scales have been studied intensely by several nonlinear optical techniques and are often accompanied by theoretical investigations. This section will discuss hole-burning experiments, followed by pump-probe and photon-echo experiments, 2D electronic experiments, and finally new theoretical approaches.

Modeling of the exciton dynamics in the BChl a chromophore complex of the FMO protein has been done using two approaches. The first describes energy transfer between chromophores by the incoherent Förster hopping rate equation, which is valid for weak coupling between the chromophores and a strong coupling of the electronic transition to vibrational states, precluding the formation of exciton levels. Excitation energy will hop from one molecule to the other along the energy gradient. However, since the existence of exciton levels in the FMO complex is well established, the Förster hopping rate equation seems not to be the most appropriate way to describe dynamics in the FMO complex. This problem was partially overcome by Iseri et al. who approximated the energy transfer rate between excitons through a linear combination of the Förster rates between the $\mathrm{BChl}$ a pigments that dominate the exciton states (Iseri and Gülen 1999). The second approach is to describe the light-induced dissipative dynamics within the framework of the multi-exciton density matrix theory. Often, the Redfield approach for the description of dissipation is used. This theory combines the time-dependent Schrödinger equation for the excitonic transitions with a linear coupling to a classical bath, given by all the vibrational modes of the chromophore complex (Renger and May 1998; Vulto et al. 1999; Brüggemann and May 2004; Brüggemann et al. 2006). Finally, a modified Redfield approach valid for intermediate coupling regimes has been applied by Read et al. (2008).

After all the light-induced coherences have vanished, the time evolution of the excitonic state populations $P_{\alpha}$, where for the FMO protein $\alpha$ runs from 1 to 7 , can be described by the Master equation (Van Amerongen et al. 2000).

$\frac{d}{d t} P_{\alpha}=\sum_{\beta} k_{\beta \rightarrow \alpha} P_{\beta}-k_{\alpha \rightarrow \beta} P_{\alpha}$,

using the rate constants $k_{\alpha \rightarrow \beta}$, which eventually lead to a thermal equilibrium within the singly excited states.

The proposed pathways of downward energy transfer are shown schematically in Fig. 4, as drawn by the respective authors. Although they show little agreement, a few general conclusions can be drawn from these results. The energy transfer from the highest to the lowest exciton level occurs on a very fast time scale; within 5 ps, mainly the lowest exciton state $P_{1}$ is populated. The population can be transferred downward either by a few big steps or by small steps including all the exciton levels.

Hole burning

Spectral dynamics, in terms of hole widths, obtained from hole-burning experiments follow a temperature dependence

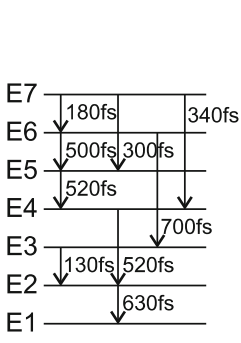

A

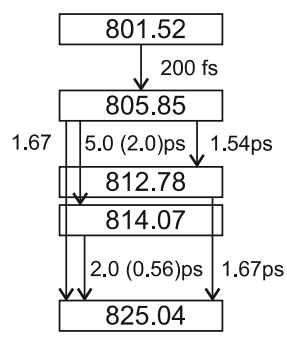

B

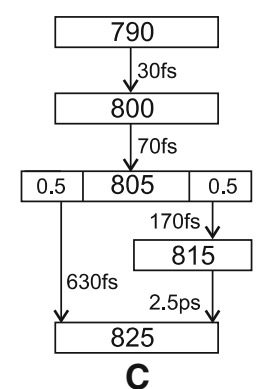

C
Fig. 4 Proposed relaxation pathways of the exciton energy in the FMO protein, with examples as given in the original references. The seven single exciton levels are represented by E1-E7. Model a from Vulto et al. (1999), b from Iseri and Gülen (1999), c from Buck et al. (1997) 
Table 8 Frequency-dependent decay times of Prosthecochloris aestuarii in Franken et al. (1998)

\begin{tabular}{ll}
\hline Wavelength $(\mathrm{nm})$ & Time constant $T_{2}$ at $6 \mathrm{~K}(\mathrm{ps})$ \\
\hline 803 & 0.5 \\
808 & 0.8 \\
811.5 & 3.1 \\
817 & 4.2 \\
820.5 & 6.0 \\
823 & 9.9 \\
826.5 & $\geq 18$ \\
829 & $\geq 19$ \\
830 & $\geq 20$ \\
\hline
\end{tabular}

power law $T^{\alpha}$, with the temperature exponent for glasses $\alpha$ $\sim 1.3$ (Matsuzaki et al. 2000). Such a power law is typical for dephasing of the excitons in a pigment coupled to a two-level system, TLS) (Yamaguchi et al. 2002). Lowfrequency excitations in a glass are often described by a TLS, modeled by a double-well potential. These excitations can contribute to the dephasing of a pigment, and hence determine the hole widths. Three states were found to contribute to the absorption band at $825 \mathrm{~nm}$. Taking into account the dephasing due to the glasslike protein, the energy transfer between the three levels within the 825-nm band occurs with 99 and 26 ps, respectively (Matsuzaki et al. 2000). Similar reasoning holds for analysis of lowenergy states in the FMO protein from Chlorobium tepidum (Rätsep et al. 1999). In order to bridge the gap between steady-state and time-resolved spectroscopy an elaborate hole-burning experiment was performed (Franken et al. 1998). On top of broad (800-820 nm) uncorrelated signals, sharp holes were detected. The observed hole widths are for an inhomogeneously broadened band twice the homogeneous linewidth, from which it is straightforward to calculate the excited state lifetimes (see Table 8). The lifetimes of the exciton states that were obtained from holeburning studies were fast, (sub)picosecond, and similar to those obtained from other methods (vide infra).

Pump-probe and photon-echo

When researchers started to study the excitation energy transfer within the FMO complex in the early 1990s, they soon realized that the dynamics occur on very fast, subpicosecond, timescales. By studying the bleach spectrum at 2 and 10 ps after excitation, it was shown that even at those short delay times, the spectrum does not exhibit a uniform bleach (Lyle and Struve 1990). In this study, the anisotropy decay was 2-4 ps. As was known from the linewidths of hole burning, the relaxation between exciton levels is complete within several hundreds of femtoseconds
(Johnson and Small 1991) and does not contribute to one color anisotropy decay. Therefore, the longer, picosecond, time constant obtained from anisotropic decay traces was attributed to hopping of excitation energy between neighboring subunits and not to lifetimes of the higher exciton states.

The obtained dephasing times from hole-burning experiments are considerably faster than values that were obtained from accumulated photon-echo experiments by Louwe and Aartsma (1994). They demonstrated that the photon-echo decay is wavelength and temperature-dependent, ranging from a decay $<1$ ps at $790 \mathrm{~nm}$ to $100 \mathrm{ps}$ at $824 \mathrm{~nm}$ at $10 \mathrm{~K}$ and exhibits multiexponential decay. They also observed a decrease of the decay times with increasing temperatures. The wavelength-dependent decay rates from the photon-echo experiments are explained on the basis of phonon-assisted dephasing, where the number of lower lying states determine the dephasing time. Initially, it was thought that the relaxation was governed by scattering within the exciton manifold. It was concluded from pumpprobe measurements that energy transfer was favored between exciton levels that lie within an energy spacing of $10 \mathrm{~nm}\left(120 \mathrm{~cm}^{-1}\right)$ (Vulto et al. 1997). At this energy, the density of acoustic phonons might be high, so that electron-phonon coupling might be the underlying mechanism of downward energy transfer. Pump-probe transients indicated a sequential relaxation of the exciton energy along a ladder of states, as was also seen in exciton simulations (Vulto et al. 1999, 1997; Buck et al. 1997; Iseri and Gülen 1999; Brüggemann and May 2004) (see Tables 9, 10, 11, 12). Figure 4 shows a couple of examples of this type of decay. Only at very low temperatures, the dephasing might be governed by downward coherent exciton transfer. The origin of the disagreement between the dephasing times from both measurements are unclear but might have to do with the distinct experimental conditions tuning into different mechanisms underlying the energy transfer in the complex.

In a more elaborate study, Louwe and Aartsma (1997) decided to take another look at the possible coherent nature of exciton transport by studying the FMO complex at $1.4 \mathrm{~K}$ with accumulated photon echoes and transient absorption

Table 9 Frequency dependent decay times of Prosthecochloris aestuarii (Vulto et al. 1997)

\begin{tabular}{lc}
\hline Wavelength (range) $(\mathrm{nm})$ & Time constants $10 \mathrm{~K}(\mathrm{ps})$ \\
\hline Blue edge & $<0.1$ \\
804 & 0.5 \\
812 & 0.17 \\
815 & 5.5 \\
823 & 37 \\
\hline
\end{tabular}


Table 10 Decay times from global analysis of pump-probe spectra of Prosthecochloris aestuariiat 19 K (Buck et al. 1997)

\begin{tabular}{ll}
\hline Number & $\tau(\mathrm{ps})$ \\
\hline 1 & 0.170 \\
2 & 0.630 \\
3 & 2.5 \\
4 & 11 \\
5 & 74 \\
6 & 840 \\
\hline
\end{tabular}

Table 11 Frequency-dependent decay times of Prosthecochloris aestuarii (Iseri and Gülen 1999)

\begin{tabular}{ll}
\hline Wavelength (range) $(\mathrm{nm})$ & Time constants-10K (ps) \\
\hline 801.52 & 0.2 \\
805.85 & $1.54,5.0(2.0)^{\mathrm{a}}, 1.67$ \\
812.78 & 1.67 \\
814.07 & $2.0(0.56)$ \\
\hline
\end{tabular}

a There was no distinct difference in the quality of the fit between the kinetic model $\mathrm{a}$ and $\mathrm{b}$ (in parenthesis)

Table 12 Lifetime of exciton states of Prosthecochloris aestuarii by exciton calculations (Brüggemann and May 2004)

\begin{tabular}{llll}
\hline Exciton number & $\tau(\mathrm{ps}) 4 \mathrm{~K}$ & $\tau(\mathrm{ps}) 77 \mathrm{~K}$ & $\tau(\mathrm{ps}) 265 \mathrm{~K}$ \\
\hline 1 & $\infty$ & 193 & 8.5 \\
2 & 82 & 33 & 3.5 \\
3 & 7.4 & 5.8 & 1.8 \\
4 & 8.8 & 6.6 & 2.0 \\
5 & 4.0 & 3.3 & 1.4 \\
6 & 2.0 & 1.9 & 1.1 \\
7 & 1.8 & 1.8 & 1.2 \\
\hline
\end{tabular}

(see Table 13). Owing to the broad exciton levels, they probed several excitonic transitions at the same time resulting in traces with multiple time constants. At long wavelengths, $(815-830 \mathrm{~nm})$ processes with exciton decay times of 5, 30, 110, and 385 ps were found, while at shorter wavelengths $(795 \mathrm{~nm})$, the decay was in the order of 100 fs. The FMO complex was described as pigments coupled to a TLS (double-well potential, in between which tunneling occurs), which is, in turn, coupled to a phonon bath. Fluctuations in the interactions between pigments due to transitions in the TLS is the main dephasing pathway in glasses below $10 \mathrm{~K}$. The TLS transitions can both influence the dipole interactions between the pigments (low frequency transitions in TLS corresponding to large displacements in the protein) as well as the site energies (high frequency, smaller displacement). At low temperatures, the coherent energy transfer is mainly limited by this coupling.
Table 13 Frequency-dependent accumulated photon echo decay times of Prosthecochloris aestuarii at $1.4 \mathrm{~K}$ (Louwe and Aartsma 1997)

\begin{tabular}{lc}
\hline$\lambda_{\max }$ of $\mathrm{DAS}^{\mathrm{a}}(\mathrm{nm})$ & Decay time $(\mathrm{ps})$ \\
\hline 827 & 385 \\
826 & 110 \\
824 & 30 \\
818 & 5 \\
\hline
\end{tabular}

a DAS spectra originate from a global analysis were the amplitudes of the different decay components are plotted against the wavelength resulting in distinct bands

Above $10 \mathrm{~K}$, the contribution of the TLS tunneling is of minor significance to the dephasing mechanism that are dominated by other processes. With these measurements, the earlier results from a preliminary study by Louwe and Aartsma (1994) were confirmed.

Several years later, interesting features were seen in low-temperature two-photon-echo (2PE) signals of both Chlorobium tepidum and Prosthecochloris aestuarii (Prokhorenko et al. 2002). At $1.27 \mathrm{~K}$, the 2PE signals show oscillations that increase in intensity when the excitation is tuned to the red edge of the absorption spectrum (up to $40 \%$ of the total amplitude for excitation at $832 \mathrm{~nm}$ ). These oscillations last up to $300 \mathrm{ps}$ and are ascribed to vibrational states of the BChl a molecule in the ground state. Fourier transforms of the 2PE traces show that the obtained frequencies match those from previous studies (Savikhin et al. 1997). In the same study, it was shown that the general theory to describe the results of photon-echo experiments did not account for the current results. The typical $\delta$ shape for dynamics in the Markov limit at initial time delays was not observed. Therefore, the dynamics were described beyond the Markov limit where system-bath memory effects occur which, among others, result in the delayed growing in of coherence in the system. At that time, it was unclear whether this had a specific function in light harvesting.

Vulto et al. used a similar approach as was used previously by Louwe et al. in the simulation of the static spectra (see "Exciton nature of the BChl a excitations in the FMO protein" and "Coupling strengths, linewidth and exciton energies"); however, to introduce dynamics, coupling of the electronic excitations to the vibrational modes in the system was included (Vulto et al. 1999). Homogeneous broadening within the system was not incorporated in the model. Owing to the weak coupling, the exciton-vibrational coupling can be treated as a perturbative term in the Hamiltonian. The distinct time scales of dephasing and the bath correlation times leads to the decoupling of the dynamics of population and coherence. The excited state dynamics, therefore, is governed by population relaxation. 
Similarly, in the simulations of Renger and May, the frequency-dependent coupling of the electronic states in the systems to the surroundings is needed. In order to describe this, the phonon-side band in a fluorescence spectrum is fitted. Using this analytical description for the spectral density, the time-resolved spectra can be fitted. As was shown before, the exciton relaxation occurs mainly between adjacent levels. The number of states lower in energy determine the relaxation rate of an exciton level. However, important additional factors are also the energy difference between the two levels and the overlap between the excitation probability densities on a single pigment $j$ (i.e., $\left|C_{\alpha}(j)\right|^{2}\left|C_{\beta}(j)\right|^{2}$ ). The authors noted that the spectra of Chlorobium tepidum fitted remarkably better than those of Prosthecochloris aestuarii, in particular an experimental decay time of 1.7 ps was not reproduced. This could be partially overcome by adjusting the site energies of especially BChl a 1 and $\mathrm{BChl}$ a 4 . The energetic order, of these pigments which are the main contributors to the second lowest exciton states (E2), seems of importance for the dynamics in the system. This was further tested by introducing inhomogeneous broadening in the system by a Monte Carlo simulation of the spectra and the dynamics. In addition to the decay time constants, distributions of time constants centered around the originally simulated values were found. At the exciton level E2, this distribution showed a clear distinction between two time domains; one of several hundreds of femtoseconds and another of several picoseconds, the latter is in the same order as the experimentally observed time scale. The spectra resulting from the Monte Carlo simulations are very similar to the dressed stick spectra calculated earlier (Vulto et al. 1998a). Vulto et al. showed that the method of Renger et al. does not reproduce the $T-S$ and LD spectra at all, and concluded that their description of the electronic structure of the FMO complex was not completely correct. However, the ingenious way of describing the spectral broadening of the transitions by Renger et al. could be used to improve future simulations.

The decay time for energy transfer from the lowest exciton state to the ground state varies widely between different techniques and research groups. Table 14 gives a clear indication that there are two timescales concerned with the lowest exciton lifetime; one of about 100 ps and a longer

Table 14 Decay times of the lowest exciton level at 77 K of Prosthecochloris aestuarii

\begin{tabular}{lc}
\hline References & $\tau$ (ns) \\
\hline Louwe and Aartsma (1997) & $0.25,3$ \\
Vulto et al. (1997) & $>0.8$ \\
Matsuzaki et al. (2000) & 2 \\
Brüggemann and May (2004) & 0.19 \\
\hline
\end{tabular}

one of several ns. A more elaborate description of this lifetime for Chlorobium tepidum is found in the electronic supplementary material. The discussion therein indicates that the lifetime of the lowest exciton state is influenced by the preparation method of the samples and in particular by the addition of oxidizing or reducing agents. However, a satisfying explanation for the latter is lacking to date.

\section{D-spectroscopy}

In the last 5 years an additional technique was used to study exciton dynamics in the FMO complex: 2D spectroscopy. This technique directly shows the frequency correlation between excited states. When there is coupling between the different states, as is the case in the FMO complex, excitation of one state influences the others. 2D electronic spectroscopy on the FMO complex is mainly used to elucidate the time-dependent couplings between exciton states. This does not provide a direct way of measuring the site energies of the individual pigments. However, in 2D electronic spectroscopy, the coupling between the exciton states will appear in the spectra directly as the so-called cross peaks (Brixner et al. 2005). In the FMO complex, the cross peaks in the 2D spectra overlap with broad and strong diagonal peaks, due to the high spectral density. In order to overcome this problem, a technique in which the diagonal peaks can be eliminated and the cross peaks are brought out was developed (Read et al. 2007). The technique is based on a scheme known from 2D-vibrational spectroscopy and uses polarization of the first two pulses to select the cross peaks. Since most of this study has been done using Chlorobium tepidum, more on this topic can be found in the electronic supplementary material.

In order to extract the contributions of the various energy decay processes in a congested 2D spectrum, polarizationdependent 2D spectroscopy was used (Read et al. 2008). In contrast to the previous study (Read et al. 2007) this was a measurement of both the rephasing and non-rephasing spectra. In the non-rephasing spectra, the diagonal linewidths of the exciton transitions are narrower and, therefore, a higher resolution can be obtained. Furthermore, the authors made use of two polarization combinations for separate $2 \mathrm{D}$ experiments. Theoretically, it is possible to obtain the projection angle $\phi$ between a pair of exciton states from the ratio between these two polarization combinations. In the nonrephasing spectra, a strong cross peak at 804 and $814 \mathrm{~nm}$ appears while changing the polarization from one to the other polarization combination. By calculating the amplitude factor of the cross peaks depending on $\phi$ for the two polarization cases, it was shown that an angle of $40^{\circ}$ reproduced the measured $2 \mathrm{D}$ data. This implies that without previous knowledge about structural properties of the system, a tentative view of the orientation of transition 
dipoles can be obtained. The current models of the FMO complex predict that excitons 2 and 4 have a high dipole strength and are the main contributors to the peaks in the spectrum at 804 and $814 \mathrm{~nm}$. Furthermore, the corresponding exciton states have a projection angle of $38^{\circ}$ verifying the results from the polarization dependent $2 \mathrm{D}$ experiments. Using the same idea of polarized fields in a theoretical study, contributions of coherent evolution and incoherent energy relaxation to a $2 \mathrm{D}$ spectrum could be separated due to a specific choice of the polarizations of the incoming pulses (Abramavicius et al. 2008b).

Currently, the best simulations of exciton dynamics are based on a method initiated by Vulto et al. (1999). An important parameter in their simulations is the coupling of an exciton state to a phonon bath. This vibronic coupling can account for energy relaxation in the FMO complex and is therefore an important factor in simulations of the exciton dynamics. In order to model the phonon-side band that mediates the coupling, they used an empirical approximation. The electron-phonon coupling was set to be equal for all states. Results of their simulations were that the exciton states preferably decay stepwise downhill along an energy gradient, as energy transfer mainly occurs between two adjacent levels. The rate of relaxation can be enhanced by the high value of the (linear) electron-phonon coupling. Cho et al. (2005) also showed that the rate of exciton transfer depends on the amplitude of the spectral density at the frequency of the transition. Using the coupling constants between the BChls of Vulto et al., except for a reduced coupling between $\mathrm{BChl}$ a 5 and 6 , the exciton dynamics were simulated using a modified Förster/Redfield theory. Rates calculated using conventional Redfield theory turned out to be too slow in the presence of weakly coupled pigments. Therefore, the weak couplings are not taken into account into the diagonalization of the Hamiltonian, but are used to calculate the rate matrix using Förster theory. Simulations of 2D electronic spectra showed a better agreement with the experiment when the modified theory was used. Adolphs et al. use an elaborate model for the spectral density by also taking into account vibrational sidebands (Adolphs and Renger 2006). In order to simulate exciton relaxation, Redfield theory was compared to the more elaborate modified theory. The latter assumed that there are possible nuclear rearrangement effects that accompany exciton relaxation. Only minor differences between the two methods were observed, where modified Redfield theory predicts slightly lower rates. Two interesting observations from their simulations are that the spectral density of the electron-phonon coupling seems optimized to dissipate excess energy during relaxation. Also, simulations revealed two different exciton relaxation branches, a slow and a fast one, which are used for energy transfer from the chlorosomes to the RC.
New theoretical approaches

As the exciton dynamics in the FMO complex is well studied and understood, a possible next step is to try and influence this dynamics. Following that route, specially shaped laser pulses are calculated using an optimal control algorithm and applied to the FMO complex (Brüggemann and May 2004). These pulses lead to a superposition of excitonic states, an excitonic wavepacket, with the target to populate just a single chromophore at a given time. The theoretical framework is given by the multi-exciton density matrix, and although the dissipation is damping the wavepacket at low temperatures, the target can be reached quite well. In a follow-up article, the additional effects of inhomogeneous broadening and orientational averaging were included (Brüggemann et al. 2006). Again, the target could be reached although to a lesser extend. The introduction of a laser field, shaped in both polarization directions, led to a larger target state population, partially working against the energetic and oriental averaging.

Under conditions encountered by the FMO complex in vivo it is very likely that multiple excitations occur within one complex. These double-excited states are more complicated than its single counterpart and are less well studied. Often 2D spectra are obscured by overlapping contributions of single and double exciton resonances. By looking at a smart representation of the $2 \mathrm{D}$ spectra using a particular set of pulses, the correlated dynamics of the double excited states can be probed (Abramavicius et al. 2008a). Strong peaks are observed for double exciton states 1,7 , and 18 that also happen to be the most delocalized states in the system. In addition, weaker signals of exciton states 9, 16, and 17 are observed. Instead of calculating the wavefunctions of the different exciton states, an alternative method can be used to describe the behavior of excitons in aggregates. In the quasiparticle approach, all the properties of the system are described in terms of scattering and double exciton energies are simply given by a sum of single exciton energies. Comparing the spectra resulting from the full calculation with that of the quasiparticle approach shows that the energies at which the peaks appear in the spectra agree, while the fine structure in the spectra of the quasiparticle approach is distorted. In order to approximate the spectra, the quasiparticle approach can be used, however, because the exciton coupling is strong, which is neglected in this approach, and the nonbosonic nature of the excitons a full calculation of the spectra is necessary for detailed analysis.

New types of 2D techniques can be developed by introducing pulse polarizations as variables into standard $2 \mathrm{D}$ schemes, as described in the previous section. This, amongst others enables the dissection of the congested 2D spectra into incoherent and coherent contributions and 
provides interesting perspective for new control strategies (Abramavicius et al. 2008b; Voronine et al. 2008).

\section{Current consensus and future directions}

Slowly the choice of parameters used to simulate the results obtained from various optical techniques is converging. Around 15-20 years ago, little agreement existed on whether to include the whole trimer or just the monomer in simulations of the spectra, on the values of the site energies and how to obtain them, and on the location of the lowest energy pigment. Nowadays, these issues seem more or less resolved: Only the monomer is taken into account in simulations, as is inhomogeneous broadening due to structural changes, BChl a 3 is principally assigned to have the lowest site energy. The parameter set from Louwe et al., including the site energies, is widely used in increasingly complex simulations. The latest addition to this is a new approach to calculate site energies instead of fitting them, using amongst others quantum chemical methods. The possible influence of the recently proposed eighth $\mathrm{BChl}$ a molecule on the variety of optical spectra could invoke new studies. It is conceivable that new detailed simulations including this pigment can lift the remaining discrepancies between experimental and theoretical spectra.

While the exact energy transfer timescales within the exciton manifold vary between techniques, it is commonly agreed that decay to the lowest exciton state occurs within several picoseconds. Despite this rapid decay, an interesting observation is the prolonged presence of coherence in the complex. This coherence with its potential role in mediating efficient energy transfer, is the topic of current research using advanced techniques such as $2 \mathrm{D}$ electronic spectroscopy and coherent control strategies with shaped excitation pulses.

Acknowledgments This study is part of the research program of the Stichting voor Fundamenteel Onderzoek der Materie (FOM), which is supported financially by the Nederlandse organisatie voor Wetenschappelijk Onderzoek (NWO).

Open Access This article is distributed under the terms of the Creative Commons Attribution Noncommercial License which permits any noncommercial use, distribution, and reproduction in any medium, provided the original author(s) and source are credited.

\section{References}

Abramavicius D, Voronine D, Mukamel S (2008a) Double-quantum resonances and exciton-scattering in coherent $2 \mathrm{D}$ spectroscopy of photosynthetic complexes. PNAS 105:8525-8530
Abramavicius D, Voronine D, Mukamel S (2008b) Unravelling coherent dynamics and energy dissipation in photosynthetic complexes by 2D spectroscopy. Biophys J 94:3613-3619

Adolphs J, Renger T (2006) How proteins trigger excitation energy transfer in the FMO complex of green sulfur bacteria. Biophys $\mathbf{J}$ 91:2778-2897

Adolphs J, Müh F, Madjet Mel-A, Renger T (2008) Calculation of pigment transition energies in the FMO protein. Photosynth Res 95:197-209

Atkins P (1995) Physical chemistry. Oxford University Press, Oxford

Ben-Shem A, Frolow F, Nelson N (2004) Evolution of photosystem I-from symmetry through pseudosymmetry to asymmetry. FEBS Lett 564(3):274-280

Brixner T, Stenger J, Vaswani H, Cho M, Blankenship R, Fleming G (2005) Two-dimensional spectroscopy of electronic couplings in photosynthesis. Nature 434:625-628

Brüggemann B, May V (2004) Ultrafast laser pulse control of exciton dynamics: a computational study on the FMO complex. J Phys Chem B 108:10529-10539

Brüggemann B, Pullerits T, May V (2006) Laser pulse control of exciton dynamics in the FMO complex: polarization shaping versus effects of structural and energetic disorder. J Photochem Photobiol A 180:322-327

Buck D, Savikhin S, Struve W (1997) Ultrafast absorption difference spectra of the Fenna-Matthews-Olson protein at 19k: experiments and simulations. Biophys J 72:24-36

Cho M, Vaswani H, Brixner T, Stenger J, Fleming G (2005) Exciton analysis in 2D electronic spectroscopy. J Phys Chem B 109:10542-10556

Fenna R, Matthews B (1975) Chlorophyll arrangement in a bacteriochlorophyll protein from Chlorobium limicola. Nature 258:573577

Francke C, Amesz J (1997) Isolation and pigment composition of the antenna system of four species of green sulfur bacteria. Photosynth Res 52:137-146

Franken E, Neerken S, Louwe RJ, Amesz J, Aartsma T (1998) A permanent hole burning study of the FMO antenna complex of the green sulfur bacterium Prosthecochloris aestuarii. Biochemistry 37:5046-5051

Gudowksa-Nowak E, Newton M, Fajer J (1990) Confromational and environmental effects on bacteriochlorophyll optical spectra: Correlations of calculated spectra with structural results. J Phys Chem 94:5795-5801

Gulbinas V, Valkunas L, Kuciauskas D, Katilius E, Liuolia V, Zhou W, Blankenship R (1996) Singlet-singlet annihilation and local heating in FMO complexes. J Phys Chem 100:17950-17956

Gülen D (1996) Interpretation of the excited-state structure of the Fenna-Matthews-Olson pigment protein complex of Prosthecochloris aestuarii based on the simultaneous simulation of the 4 $\mathrm{k}$ absorption, linear dichroism and singlet-triplet asborption difference spectra: a possible excitonic explanation? J Phys Chem 100:17683-17689

Hayes JM, Ruehlaender M, Soukoulis CM, Small GJ (2002) Monte carlo simulations of energy transfer rates: application to downward energy transfer within the $825 \mathrm{~nm}$ absorption band of the FMO complex of Prosthecochloris aestuarii. J Lumin 98:246-255

Iseri E, Gülen D (1999) Electronic excited states and excitation transfer kinetics in the Fenna-Matthews-Olson protein of the photosynthetic bacterium Prosthecochloris aestuarii at low temperatures. Eur Biophys J 28:243-253

Johnson S, Small G (1991) Excited-state structure and energy-transfer dynamics of the bacteriochlorophyll $a$ antenna complex from Prosthecochloris aestuarii. J Phys Chem 95:471-479 
Li Y, Zhou W, Blankenship R, Allen J (1997) Crystal structure of the bacteriochlorophyll $a$ protein from Chlorobium tepidum. J Mol Biol 271:456-471

Louwe R, Aartsma T (1994) Optical dephasing and excited state dynamics in photosynthestic pigment-protein complexes. J Lumin 58:154-157

Louwe R, Aartsma T (1997) On the nature of energy transfer at low temperatures in the bchl $a$ pigment-protein complex of green sulfur bacteria. J Phys Chem B 101:7221-7226

Louwe R, Vrieze J, Aartsma T, Hoff A (1997a) Toward an integral interpretation of the optical steady-state spectra of the FMOcomplex of Prosthecochloris aestuarii. 1. an investigation with linear-dichroic absorbance-detected magnetic resonance. J Phys Chem B 101:11273-11279

Louwe R, Vrieze J, Hoff A, Aartsma T (1997b) Toward an integral interpretation of the optical steady-state spectra of the FMOcomplex of Prosthecochloris aestuarii. 2. exciton simulations. J Phys Chem B 101:11280-11287

Lu X, Pearlstein R (1993) Simulations of Prostechochloris bacterioschlorophyll a protein optical spectra improved by parametric computer search. Photochem Photobiol 57:86-91

Lyle P, Struve W (1990) Evidence for ultrafast exciton localization in the $Q_{y}$ band of bacteriochlorophyll a -protein from Prosthecochloris aestuarii. J Phys Chem 94:7338-7339

Matsuzaki S, Zazubovich V, Rätsep M, Haynes J, Small G (2000) Energy transfer kinetics and low energy vibrational structure of the three lowest energy $Q_{y}$-states of the Fenna-Matthews-Olson antenna complex. J Phys Chem B 104:9564-9572

Matthews B, Fenna R, Bolognesi MC, Schmid MF, Olson JM (1979) Structure of a bacteriochlorophyll a-protein from the green photosynthetic bacterium Prosthecochloris aestuarii. J Mol Biol 25:259-285

May V, Kühn O (2000) Charge and energy transfer dynamics in molecular systems. Wiley-VCH, Berlin

Melkozernov A, Olson J, Li YF, Allen J, Blankenship R (1998) Orientation and excitonic interactions of the Fenna-MatthewsOlson bacteriochlorophyll $a$ protein in membranes of the green sulfut bacterium Chlorobium tepidum. Photosynth Res 56:315-328

Müh F, Madjet M, Adolphs J, Abdurahman A, Rabenstein B, Ishikita H, Knapp EW, Renger T (2007) Alpha-helices direct excitation energy flow in the Fenna-Matthews-Olson protein. PNAS 104:16862-16867

Olson J (2004) The FMO protein. Photosynth Res 80:181-187

Olson J, Romano C (1962) A new chlorophyll from green bacteria. Biochim Biophys Acta 59:726-728

Olson J, Ke B, Thompson K (1976) Exciton interactions among chlorophyll molecules in bacteriochlorophyll a proteins and bacteriochlorophyll a reaction center complexes from green bacteria. Biochim Biophys Acta 430:524-537

Pearlstein R (1992) Theory of the optical spctra of the bacteriochlorophyll $a$ antenna protein trimer from Prosthecochloris aestuarii. Photosynth Res 31:213-226

Prokhorenko V, Holzwarth A, Nowak F, Aartsma T (2002) Growingin of optical coherence in the FMO antenna complexes. J Phys Chem B 106:9923-9933

Rätsep M, Freiberg A (2007) Unusual temperature quenching of bacteriochlorophyll $a$ fluorescence in FMO antenna protein trimers. Chem Phys Lett 434:306-311

Rätsep M, Wu HM, Hayes J, Blankenship R, Cogdell R, Small G (1998) Stark hole-burning studies of three photosynthetic complexes. J Phys Chem B 102:4035-4044

Rätsep M, Blankenship R, Small G (1999) Energy transfer and spectral dynamics of the three lowest energy $Q_{y}$-states of the Fenna-Matthews-Olson antenna complex. J Phys Chem B 103:5736-5741
Read E, Engel G, Calhoun T, Mančal T, Ahn TK, Blankenship RE, Fleming GR (2007) Cross-peak-specific two-dimensional electronic spectroscopy. PNAS 104:14203-14208

Read E, Schlau-Cohen G, Engel G, Wen J, Blankenship R, Fleming $\mathrm{G}$ (2008) Visualization of excitonic structure in the fennamatthews-olson photosynthetic complex by polarization-dependent two-dimensional electronic spectroscopy. Biophys J 95:847-856

Rémigy HW, Stahlberg H, Fotiadis D, Müller S, Wolpensinger B, Engel A, Hauska G, Tsiotis G (1999) The reaction center complex from the green sulfur bacterium Chlorobium tepidum: a structural analysis by scanning transmission electron microscopy. J Mol Biol 290:851-858

Renger T, May V (1998) Ultrafast exciton motion in photosynthetic antenna systems: the FMO complex. J Phys Chem A 102:43814391

Savikhin S, Buck D, Struve W (1997) Oscillating anisotropies in a bacteriochlorophyll protein: evidence for quantum beating between exciton levels. Chem Phys 223:303-312

Tronrud D, Schmid M, Matthews B (1986) Structure and x-ray amino acid sequence of a bacteriochlorophyll a protein from Prosthecochloris aestuarii refined at $1.9 \AA$ resolution. J Mol Biol 188:443-454

Tronrud DE, Wen J, Gay L, Blankenship RE (2009) The structural basis for the difference in absorbance spectra for the FMO antenna protein from various green sulfur bacteria. Photosynth Res 100(2):79-87

Van Amerongen H, Valkunas L, Van Grondelle R (2000) Photosynthetic excitons. World Scientific Publishing, Singapore

Van Mourik F, Verwijst R, Mulder J, Van Grondelle R (1994) Singlet-triplet spectroscopy of the light-harvesting bchl $a$ complex of Prosthecochloris aestuarii: the nature of the low-energy $825 \mathrm{~nm}$ transition. J Phys Chem 98:10307-10312

Voronine D, Abramavicius D, Mukamel S (2008) Chirality-based signatures of local protein environments in two-dimensional optical spesctroscopy of two species photosynthetic complexes of green sulfur bacteria: simulation study. Biophys J 95:48964907

Vulto S, Streltsov A, Aartsma T (1997) Excited state energy relaxation in the FMO complexes of the green bacterium Prostecochloris aestuarii at low temperatures. J Phys Chem B 101:4845-4850

Vulto S, De Baat M, Louwe R, Permentier H, Neef T, Miller M, Van Amerongen H, Aartsma T (1998a) Exciton simulations of optical spectra of the FMO complex from the green sulfer bacterium Chlorobium tepidum at 6k. J Phys Chem B 102:9577-9582

Vulto S, Neerken S, Louwe R, De Baat M, Amesz J, Aartsma T (1998b) Excited-state structure and dynamics in FMO antenna complexes from photosynthetic green sulfur bacteria. J Phys Chem B 102:10630-10635

Vulto S, De Baat M, Neerken S, Nowak F, Van Amerongen H, Amesz J, Aartsma T (1999) Excited state dynamics in FMO antenna complexes from photosynthetic green sulfur bacteria: a kinetic model. J Phys Chem B 103:8153-8161

Wen J, Zhang H, Gross M, Blankenship R (2009) Membrane orientation of the fmo antenna protein from Chlorobaculum tepidum as determined by mass spectrometry-based footprinting. PNAS 106:6134-6139

Wendling M, Pullerits T, Przyjalgowski M, Vulto S, Aartsma T, Van Grondelle R, Van Amerongen H (2000) Electron-vibrational coupling in the Fenna-Matthews-Olson complex of Prosthecochloris aestuarii determined by temperature-dependent absorption and fluorescence line-narrowing measurements. J Phys Chem B 104:5825-5831 
Wendling M, Przyjalgowski M, Gülen D, Vulto S, Aarstma T, Van Grondelle R, Van Amerongen H (2002) The quantative relationship between structure and polarized spectroscopy in the FMO complex of Prosthecochloris aestuarii: refining experiments and simulations. Photosynth Res 71:99-123
Yamaguchi M, McIntire M, Chronister E (2002) A photon echo study of two-level systems in polyisobutylene under high pressure. J Chem Phys 116:1737-1743 